\title{
Understanding Galactic planetary nebulae with precise/reliable nebular abundances
}

\author{
D. A. García-Hernández ${ }^{1,2}$, P. Ventura ${ }^{3}$, G. Delgado-Inglada ${ }^{4}$, \\ F. Dell'Agli ${ }^{1,2,3}$, M. Di Criscienzo ${ }^{3}$ and A. Yagüe . $^{1,2,3}$ \\ ${ }^{1}$ Instituto de Astrofísica de Canarias, C/ Vía Láctea s/n, E-38205 La Laguna, Spain \\ ${ }^{2}$ Departamento de Astrofísica, Universidad de La Laguna, E-38206 La Laguna, Spain \\ ${ }^{3}$ INAF - OAR, Via Frascati 33, 00040, Monte Porzio Catone, Italy \\ ${ }^{4}$ Instituto de Astronomía, UNAM, Apdo. Postal 70264, 04510, México D. F., México
}

\begin{abstract}
We compare recent precise/reliable nebular abundances - as derived from high-quality optical spectra and the most recent ICFs - in a sample of Galactic planetary nebulae (PNe) with nucleosynthesis predictions $(\mathrm{HeCNOCl})$ from asymptotic giant branch (AGB) ATON models in the metallicity range $Z_{\odot} / 4<Z<2 Z_{\odot}$. According to the infrared dust features, the sample is divided among carbon-, oxygen-, and double-dust chemistry (CC, OC, and DC, respectively), providing an independent proxy for the nature of the PNe progenitors. Our AGB models, with diffusive overshooting from all the convective borders, nicely reproduce the $\mathrm{O}$ overabundances observed in $\mathrm{CC} \mathrm{PNe}$, indicating that they evolve from low-Z low-mass $\left(\sim 1-3 \mathrm{M}_{\odot}\right)$ AGB stars. This indicates that $\mathrm{O}$ is not always a good indicator of the original ISM metallicity and that the $\mathrm{O}$ production by low-mass stars should be considered in galactic-evolution models. The lowest metallicity OC PNe evolve from low-mass $\left(\sim 1 \mathrm{M}_{\odot}\right)$ O-rich AGBs, while the higher metallicity ones (all with uncertain dust classifications) display a chemical pattern similar to the DC PNe. In agreement with the recent literature, the DC PNe mostly descend from high-mass $(\mathrm{M}>3.5$ $\mathrm{M}_{\odot}$ ) solar/supersolar metallicity AGBs that experience hot bottom burning (HBB), but other formation channels in low-mass AGBs like extra mixing, stellar rotation, binary interaction, or He pre-enrichment cannot be disregarded until more accurate $\mathrm{C} / \mathrm{O}$ ratios can be obtained. Two DC PNe show the imprint of advanced CNO processing and deep second dredge-up, suggesting progenitors masses close to the limit to evolve as core collapse supernovae (above $6 \mathrm{M}_{\odot}$ ). Their actual $\mathrm{C} / \mathrm{O}$ ratios, if confirmed, indicate contamination from the third dredge-up, rejecting the hypothesis that the chemical composition of such high-metallicity massive AGBs is modified exclusively by HBB.
\end{abstract}

Keywords. Nuclear reactions, nucleosynthesis, abundances, ISM: abundances, H II regions, planetary nebulae: general, Galaxy: abundances.

\section{Introduction}

The chemical composition of planetary nebulae (PNe) proves an extremely useful and valuable tool to constrain asymptotic giant branch (AGB) models (see e.g., Ventura et al. 2015). The chemistry of $\mathrm{PNe}$ is the outcome of the combination of the different processes that contribute to alter the surface chemical composition during the whole AGB life. In addition, PNe - because of their emission-line nature - are easily observed at very large distances, and the gas chemical composition can be derived. Some chemical elements (e.g., $\mathrm{Ar}$ and $\mathrm{Cl}$ ) may remain practically unchanged in PNe, reflecting the primordial composition of the interstellar medium (ISM) where their progenitor stars were born. Other elements like $\mathrm{C}$ and $\mathrm{N}$ (to a lesser extent $\mathrm{O}$ ), however, may be strongly modified during the previous AGB phase. The $\mathrm{O}$ abundance in $\mathrm{PNe}$ has been widely used as a metallicity indicator of the ISM where they originated; e.g., to derive metallicity 
gradients in our own Galaxy and other nearby galaxies (see e.g., Richer 2015). This is because "standard" (with no extra-mixing processes) nucleosynthesis theoretical models of low- and intermediate-mass stars available in the literature (e.g., Karakas 2010) do not predict significant $\mathrm{O}$ enrichment (or destruction) at near-solar metallicities during the AGB phase.

However, by using high-quality optical spectra in conjunction with the best available ionization correction factors (ICFs), Delgado-Inglada et al. (2015) have very recently reported the first observational evidence of $\mathrm{O}$ self-enrichment (by $\sim 0.3$ dex) in Galactic PNe with C-rich dust; the expected outcome of low-mass $\left(\sim 1.5-3 \mathrm{M}_{\odot}\right)$ stars. Models that include diffusive convective overshooting (e.g., Herwig et al. 1997)† predict a significant production of oxygen, even at solar metallicities. This mixing process in combination with an efficient third dredge-up (TDU), may produce an increase of $\mathrm{O}$ abundance for low-mass $\left(\sim 1.5-3 \mathrm{M}_{\odot}\right)$ stars. Rotation, magnetic fields, and thermohaline mixing are alternative mechanisms, even less understood, that may cause extra-mixing (see e.g., Karakas \& Lattanzio 2014 for a review). Here we present the main results from the comparison of self-consistent AGB nucleosynthesis predictions (based on the ATON code) for the CNO elements and $\mathrm{He}$ in the metallicity range $Z_{\odot} / 4<Z<2 Z_{\odot}$ with the Delgado-Inglada et al. (2015) sample of Galactic PNe (see García-Hernández et al. 2016a,b).

\section{The AGB ATON models}

Briefly, the AGB ATON models are calculated using the following physical ingredients: (i) The convection is modelled according to the full spectrum of turbulence (FST) model. In regions unstable to convection, mixing of chemical and nuclear burning are coupled by a diffusion-like equation. Overshoot of convective eddies into the radiatively stable regions follows an exponential decay of velocities from the border of the convective zones (which is fixed via the Schwarzschild criterion) with the e-folding distance of the decay given by $\zeta H_{p}=0.002$; (ii) Different mass-loss prescriptions are used for O- and C-rich AGB stars; (iii) The molecular opacities at low temperatures $\left(<10^{4} \mathrm{~K}\right)$ are calculated with the AESOPUS tool (Marigo \& Aringer 2009), which is especially important for the description of the C-rich phase. We refer the reader to García-Hernández et al. (2016a,b) for more details about the AGB ATON models as well as for an overview of the AGB ATON abundances predictions.

\section{Understanding the Galactic PNe sample with precise/reliable nebular abundances}

Fig. 1 compares the Delgado-Inglada et al. (2015) sub-sample (7 objects) of lower metallicity Galactic PNe with C-dust features in their mid-IR spectra (García-Hernández et al. (2016a). By comparing the observed chemistry ( $\mathrm{HeCNOCl}$ ) with the AGB ATON models, García-Hernández et al. (2016a) concluded that they mostly descend from lowmetallicity $\left(Z=8 \times 10^{-3}\right)$ low-mass $\left(\sim 1-3 \mathrm{M}_{\odot}\right)$ AGB stars that produce O, confirming that $\mathrm{O}$ is not always a good metallicity indicator (especially for low-metallicity PNe with C-rich dust) and that $\mathrm{O}$ production by low-mass stars should be thus considered in the Galactic chemical evolution models.

On the other hand, Fig. 2 compares the Delgado-Inglada et al. (2015) sub-samples (13 objects) of Galactic PNe with O-rich and/or C-rich dust features in their mid-IR spectra

$\dagger$ The Pignatari et al. (2016) nucleosynthesis models (only very recently accepted for publication) also predict $\mathrm{O}$ production in low-mass stars. 

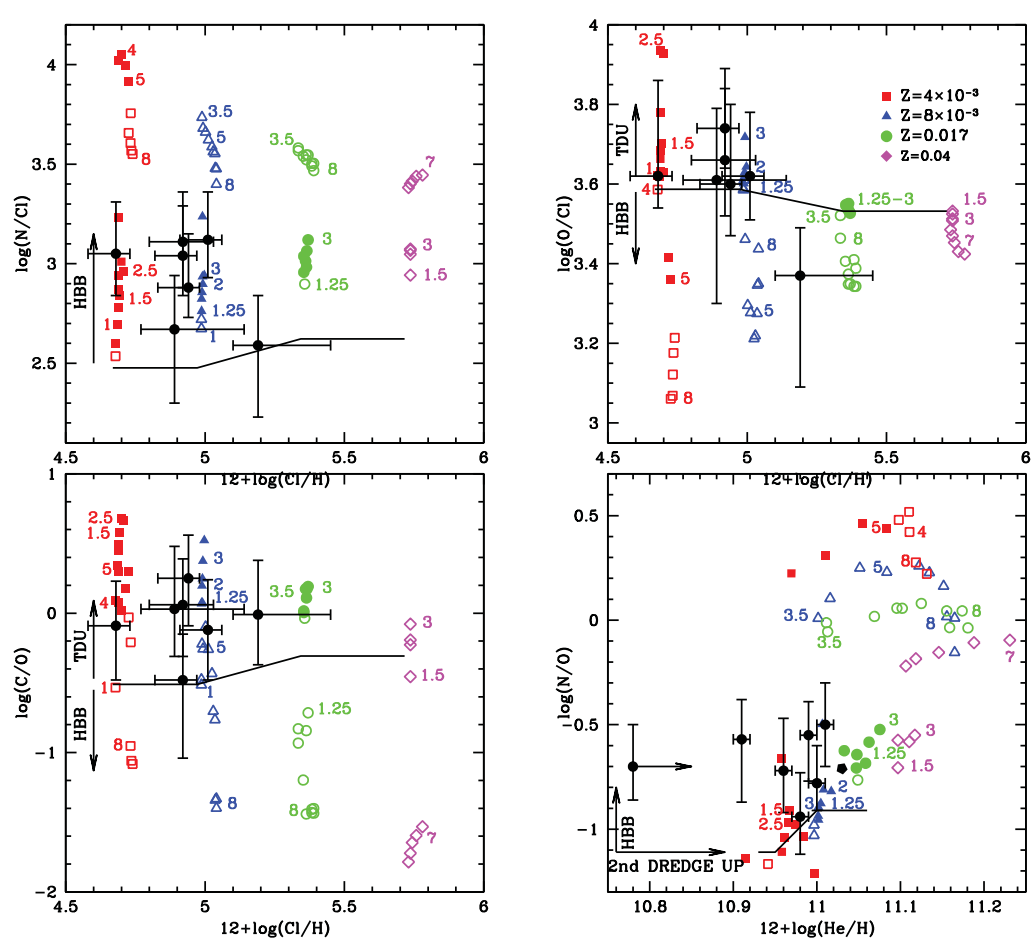

Figure 1. Chemical abundances of Galactic carbon-dust PNe (solid circles, Delgado-Inglada et al. 2015) vs. the AGB ATON model predictions for different masses (a few relevant masses are marked) and metallicities: $\mathrm{Cl}$ vs. $\mathrm{N} / \mathrm{Cl}$ (top, left), $\mathrm{Cl}$ vs. $\mathrm{O} / \mathrm{Cl}$ (top, right), $\mathrm{Cl}$ vs. $\mathrm{C} / \mathrm{O}$ (bottom, left), He vs. N/O (bottom, right). The thin, solid lines indicate the assumed initial abundances, while the arrows indicate the qualitative effects of the HBB, SDU and TDU. Filled and open symbols correspond to C- and O-rich stars, respectively. Updated from García-Hernández et al. (2016a).

(García-Hernández et al. 2016b). Unlike the Delgado-Inglada et al. (2015) subsample of carbon-dust chemistry (CC) PNe (García-Hernández et al. 2016a), here the individual abundance errors (in particular that of the $\mathrm{C} / \mathrm{O}$ ratio), the wide metallicity range, and the uncertain dust types/subtypes in some objects do not permit a clear determination of the AGB initial mass for both PNe samples (see García-Hernández et al. 2016b for a full discussion). The PNe observations versus AGB models comparison is thus more focussed on a star-by-star basis. By comparing the models vs. observations (Fig. 2), García-Hernández et al. (2016b) concluded that: i) The two lowest metallicity OC PNe are interpreted as the descendants of low-mass $\left(\sim 1 \mathrm{M}_{\odot}\right)$ AGB stars that did not reach the C-rich phase, while the three PNe of higher metallicities in this group have uncertain dust classifications and are chemically indistinguishable from the rest of DC PNe in our sample, being interpreted as truly DC PNe; ii) The DC PNe in our sample are best separated (in terms of progenitor AGB masses) in the He vs. N/O plane, which otherwise suggests that they mostly descend from high mass $\left(M \geqslant 3.5 M_{\odot}\right)$ HBB AGB stars of solar/supersolar metallicities. This is consistent with the recent work in the literature, and also we cannot discard alternative formation channels in low-mass non-HBB stars such as extra mixing, stellar rotation, binary interaction, or He pre-enrichment (more precise $\mathrm{C} / \mathrm{O}$ ratios turn out to be fundamental to learn about the stellar origin of DC $\mathrm{PNe}$ ); iii) Two DC PNe are likely descendants of the more massive AGB stars of close-tosolar chemistry, with masses $\sim 6-7 M_{\odot}$. The $\mathrm{C} / \mathrm{O}$ ratios of these objects $(C / O \sim 0.5)$, 

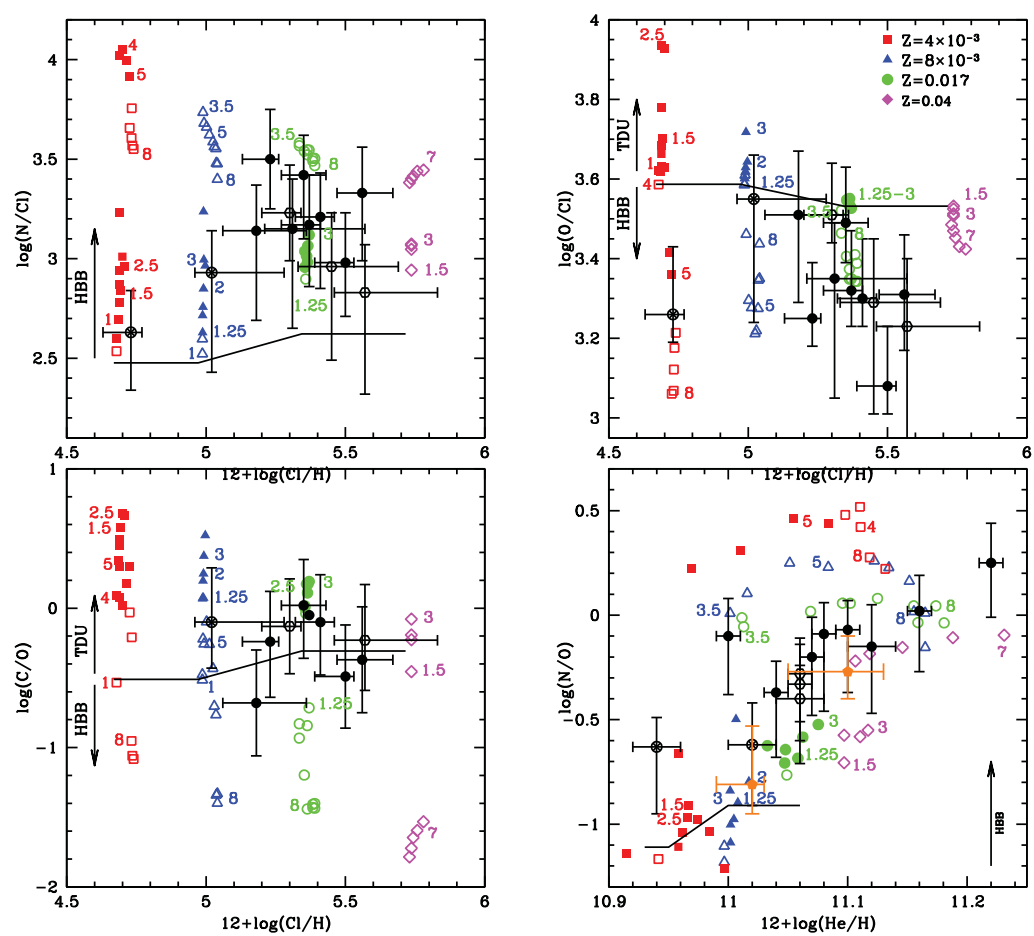

Figure 2. Same as Fig. 1 but for the Galactic OC (open-crossed circles) and DC (solid circles) PNe of Delgado-Inglada et al. (2015). The three OC PNe with more uncertain dust classifications (see García-Hernández et al. 2016b for more details) are shown with open hexagons. Updated from García-Hernández et al. (2016b).

if confirmed, suggests a role of the TDU in the contamination of the surface chemistry during the previous AGB phase, being at odds with our AGB model predictions.

\section{Acknowledgments}

DAGH acknowledges support provided by the RYC-2013-14182 fellowship and the MINECO AYA-2014-58082-P grant.

\section{References}

Delgado-Inglada, G., Rodríguez, M., Peimbert, M. et al. 2015, MNRAS, 449, 1797

García-Hernández, D. A., Ventura, P., Delgado-Inglada, G. et al. 2016a, MNRAS, 458, L118

García-Hernández, D. A., Ventura, P., Delgado-Inglada, G. et al. 2016b, MNRAS, 461, 542

Herwig, F., Blöcker, T., Schönberner, D., El Eid, \& M. 1997, A\& A, 324, L81

Karakas A. I. 2010, MNRAS, 403, 1413

Karakas A. I., Lattanzio J. C. 2014, PASA, 31, 30

Marigo P. \& Aringer B. 2009, A\& A, 508, 1538

Pignatari, M., Herwig, F., Hirschi, R., et al. 2016, ApJS, 225, 24

Ventura P., Stanghellini, L., Dell'Agli, F. et al. 2015, MNRAS, 452, 3679

Richer, M. G. 2015, in IAU General Assembly, Meeting \#29, \#2256079 